\title{
Improvement of thermoelectric properties of composite films of PEDOT-PSS with xylitol by means of stretching and solvent treatment
}

\author{
Shoko Ichikawa ${ }^{1}$ and Naoki Toshima ${ }^{1,2}$ \\ Organic thermoelectric materials have advantages, such as their use in the easy and low-cost fabrication of flexible devices \\ with a large area that can convert waste heat energy into electricity and vice versa. The critical point of the materials is \\ their electroconductivity and flexibility for practical applications. Stiff and brittle poly(3,4-ethylenedioxythiophene) \\ poly(4-styrenesulfonate) films are known to become flexible by forming composites with xylitol. Thus, we prepared flexible \\ poly(3,4-ethylenedioxythiophene) poly(4-styrenesulfonate)/xylitol composite films and evaluated their thermoelectric properties. \\ Stretching the composite films, fabricating composite films by adding dimethyl sulfoxide, and treating the films with dimethyl \\ sulfoxide and/or pure water were found to increase the electrical conductivity and improve the thermoelectric properties of \\ the films.
}

Polymer Journal (2015) 47, 522-526; doi:10.1038/pj.2015.28; published online 13 May 2015

\section{INTRODUCTION}

The 11th March disaster in 2011 in the Tohoku region, Japan, and the subsequent accident at the Fukushima Dai-ichi nuclear power station highlighted the importance of saving energy and developing a new and eco-friendly form of energy. Converting low-temperature waste heat into electricity is helpful for saving energy in general. ${ }^{1-3}$ Since their discovery in $1977^{4}$, conducting polymers have been applied to organic electronic devices as actuators, condensers, electrodes, and for other purposes because of their advantages such as flexibility, light weight, easy processing and environmentally friendly properties. ${ }^{5}$ Poly(3,4-ethylenedioxythiophene) poly(4-styrenesulfonate $)^{6}$ is one of the most useful materials among the many conducting polymers that have been synthesized for decades because it is highly electroconductive and is commercially available as an aqueous dispersion.

Recently, organic thermoelectric materials have received much attention for their conversion of heat energy (temperature difference) into electricity and vice versa. ${ }^{1-3}$ The critical point of the organic thermoelectric materials is their electroconductivity and flexibility when they are used as films in practical devices. We started research into the organic thermoelectric properties of conducting polymers in the late 1990s by using conducting polyaniline ${ }^{7}$ and found that their low thermal conductivity is one of the most important advantages of organic thermoelectronics. ${ }^{8}$

It is well known that stretching conducting films causes polymer chain orientation, which increases the electrical conductivity $(\sigma)$ of the film. ${ }^{9}$ We reported that stretching polyaniline films increased the electrical conductivity and resulted in an improvement of the thermoelectric performance. ${ }^{10}$ The reason can be clarified by equation (1), where $e, n$ and $\mu$ are the electrical charge of the carrier, carrier concentration and carrier mobility, respectively. ${ }^{11}$ The equation indicates that the electrical conductivity $(\sigma)$ is proportional to the carrier mobility $(\mu)$, which may increase with the increasing orientation of the polymer chains by stretching. This increase in the electrical conductivity can lead to an increasing figure-of-merit $(Z T)$, as obtained using equation (2), in which $S, T$ and $\kappa$ are the Seebeck coefficient, absolute temperature and thermal conductivity, respectively. ${ }^{11}$ The Seebeck coefficient can be characterized by equation (3), where $m^{*}, k$ and $h$ are the effective mass of the carrier, Boltzmann constant $\left(1.38 \times 10^{-23} \mathrm{JK}^{-1}\right)$ and Planck constant $\left(6.63 \times 10^{-34}\right)$, respectively. ${ }^{11}$ The thermal conductivity, $k$, can be calculated as the product of the thermal diffusivity $(\alpha)$, density $(\rho)$ and specific heat $\left(C_{\mathrm{p}}\right)$ as shown in equation (4). ${ }^{11}$ We have successfully prepared highly electroconductive polyphenylenevinylene derivatives by using the stretching technique, which resulted in organic thermoelectric materials with $Z T=0.1 .^{12}$

$$
\begin{aligned}
& \sigma=e n \mu \\
& Z T=S^{2} \sigma T / \kappa \\
& S=\frac{\pi^{2} k^{2} m^{*} T}{\left(3 \pi^{2}\right)^{2 / 3} h e n^{2 / 3}} \\
& \kappa=\alpha \rho C_{\mathrm{p}}
\end{aligned}
$$

${ }^{1}$ Division of Materials for Energy Conversion, Advanced Materials Institute, Tokyo University of Science Yamaguchi, Yamaguchi, Japan and ${ }^{2}$ Department of Applied Chemistry, Tokyo University of Science Yamaguchi, Yamaguchi, Japan

Correspondence: Professor N Toshima, Department of Applied Chemistry, Tokyo University of Science Yamaguchi, Daigaku-dori, SanyoOnoda-shi, Yamaguchi 756-0884, Japan E-mail: toshima@rs.tus.ac.jp

Received 10 January 2015; revised 24 February 2015; accepted 11 March 2015; published online 13 May 2015 
The improvement of thermoelectric performance (increase of $Z T$ value) can be achieved by increasing $S$ and $\sigma$ and by decreasing $\kappa$ according to equation (2). The $S$ value can be enhanced by increasing $m^{*}$ and decreasing $n$, according to equation (3). The $m^{*}$ value is determined by the type of material. A decrease of the carrier concentration $n$ decreases the electrical conductivity $\sigma$ at the same time, according to equation (1). Conversely, an increase in carrier mobility $\mu$ can directly increase the electrical conductivity $\sigma$ according to equation (1), and has no direct effect on the Seebeck coefficient $S$ and thermal conductivity $\kappa$. Thus, the stretching technique is expected to provide an excellent method to improve the $Z T$ value by increasing the carrier mobility $\mu$.

It was recently reported that PEDOT-PSS films, which were stiff and brittle by themselves, became flexible by forming composites with xylitol and that the stretched composite films had higher electrical conductivity than the pristine films did before the stretching. ${ }^{13}$

In addition, it is well known that both preparing films by casting a PEDOT-PSS aqueous solution containing dimethyl sulfoxide $(\mathrm{DMSO})^{14-16}$ and rinsing PEDOT-PSS films with pure water ${ }^{17} \mathrm{can}$ improve the electrical conductivity of the films.

In the present paper, we first report the preparation of flexible composite PEDOT-PSS films containing xylitol and the evaluation of their thermoelectric properties. In addition, stretching the composite films, preparing the composite films by adding DMSO and treating the films with pure water were carried out to increase the electrical conductivity and improve the thermoelectric properties of the films.

\section{EXPERIMENTAL PROCEDURE}

\section{Film preparation}

The PEDOT-PSS films were prepared by casting a commercial dispersion of PEDOT-PSS in water (Clevious PH1000, Heraeus Precious Metals GmbH \& Co. KG, Leverkusen, Germany, 1 wt.\%) on a quartz substrate and drying at $40-$ $50^{\circ} \mathrm{C}$ for $15 \mathrm{~h}$ and then at $110^{\circ} \mathrm{C}$ for $2 \mathrm{~h}$ in a vacuum oven.

To prepare the free-standing flexible composite films of PEDOT-PSS and xylitol, a composite dispersion was prepared by adding xylitol powder (Sigma-Aldrich Co., St Louis, MO, USA, 50 wt.\% of poly(3,4-ethylenedioxythiophene) poly(4-styrenesulfonate)) to the PEDOT-PSS dispersion and stirring the mixture with a magnetic stirrer for $1 \mathrm{~h}$. The dispersion was put into a Teflon petri dish and then dried on a hot plate at $50{ }^{\circ} \mathrm{C}$ for $\sim 12 \mathrm{~h}$ in air to produce the thick cast composite films.

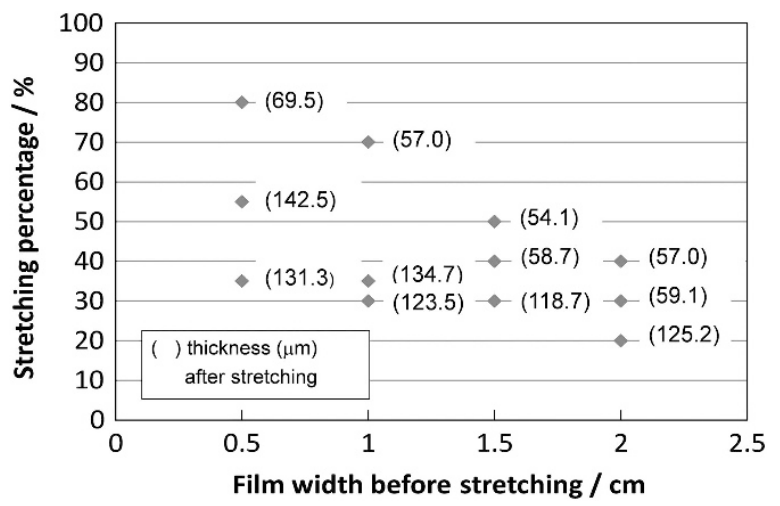

Figure 1 Relation between the film width before stretching and the stretching percentage after stretching PEDOT-PSS films containing $50 \mathrm{wt} . \%$ xylitol of PEDOT-PSS. A full color version of this figure is available at Polymer Journal online.

\section{Stretching of films}

The film, which was $50-150 \mu \mathrm{m}$ thick, $6.0 \mathrm{~cm}$ long and $0.5,1.0,1.5$ or $2.0 \mathrm{~cm}$ wide, was held with both hands at both edges of the film and stretched under controlled heating at $\sim 60^{\circ} \mathrm{C}$ on a home-made electric heater. The stretched films were then annealed on a hot plate at $140^{\circ} \mathrm{C}$ for $1 \mathrm{~h}$. The stretched films thus prepared were plastic under ordinary conditions and could not be repeatedly stretched at all. The stretching ratio was calculated as a percent by the lengths before and after stretching.

\section{DMSO addition}

PEDOT-PSS(DMSO) and PEDOT-PSS(DMSO+xylitol) films were prepared by casting the dispersion prepared by adding only 5 wt.\% of DMSO (SigmaAldrich) and both $5 \mathrm{wt} . \%$ of DMSO and $50 \mathrm{wt} . \%$ of xylitol to the PEDOT-PSS dispersions. All of the films were dried on a hot plate at $40-50^{\circ} \mathrm{C}$ for $12 \mathrm{~h}$. The PEDOT-PSS(DMSO) films were further heated at $110^{\circ} \mathrm{C}$ for $2 \mathrm{~h}$ in vacuum, and the PEDOT-PSS(DMSO+xylitol) films were heated at $140^{\circ} \mathrm{C}$ for $1 \mathrm{~h}$ on a hot plate in air.

\section{DMSO and water treatment of the films}

Instead of adding DMSO to the PEDOT-PSS aqueous solution and casting the solution to form the films, the dried films were soaked in DMSO for $1 \mathrm{~h}$ at room temperature, removed from the solution and dried by heating at $140{ }^{\circ} \mathrm{C}$ for $1 \mathrm{~h}$ on a hot plate in air.

For the water treatment, the surfaces of the PEDOT-PSS(xylitol) films were covered by a small amount of pure water for $30 \mathrm{~min}$. After removing the water from the surface with a pipette, the films were dried at $140^{\circ} \mathrm{C}$ for $1 \mathrm{~h}$ on a hot plate in air.

\section{Measurement of the thermoelectric properties}

The Seebeck coefficients and electrical conductivity were measured at least two times with a ULVAC ZEM-3 M8 instrument (ULVAC-RIKO Inc., Yokohama, Kanagawa, Japan) at $330-430 \mathrm{~K}$ under vacuum with $\mathrm{He}$ or with Lorester (Mitsubishi Chemical Analytech Co. Ltd, Chigasaki, Kanagawa, Japan, for electrical conductivity) at room temperature. X-ray diffraction spectra of these films were measured by a Rigaku Corporation RINT-TTRIII X-ray diffractometer (Rigaku Corp., Akishima, Tokyo, Japan) using a CuKo ray at the UBE Science Analysis Laboratory, Inc, Ube, Yamaguchi, Japan. The measurement of the thermal diffusivity $(\kappa)$ was carried out with a Netzsch LFA 447 Nanoflash Xe flash analyzer (Netzsch Japan Ltd, Yokohama, Kanagawa, Japan), the density $(\rho)$ was calculated by the volume and mass, and the specific heat $\left(C_{\mathrm{p}}\right)$ was measured using a Netzsch DSC F1 Phoenix instrument (Netzsch Japan Ltd) at $273-423 \mathrm{~K}$

\section{RESULTS AND DISCUSSION}

\section{Stretching characteristics}

Although the PEDOT-PSS films were stiff and brittle, the composite films of PEDOT-PSS(xylitol) were flexible and soft. Thus, the composite films could be stretched by applying a force in the direction parallel to the plane at $\sim 60^{\circ} \mathrm{C}$. The color of films was the same before and after the stretching. Although the stretched films became wavy, they kept their shape and size under ordinary conditions and became slightly stronger than the pristine films. Because the stretching was carried out by hand, the narrow films were more easily stretched than the wide films were. The width of the pristine PEDOT-PSS(xylitol) composite films for stretching was observed to be inversely proportional to the stretching percentage of the stretched film, as shown in Figure 1.

The higher the stretching percentage of the films was the thinner the thickness was compared with the films that had the same film width. The stretching percentage of films that were $0.5 \mathrm{~cm}$ in width was two times as great as those that were $2.0 \mathrm{~cm}$ in width.

The thermoelectric properties of the stretched composite films are shown in Figures 2, 3 and 4. First, the narrower the film width, the higher the electrical conductivity was. In addition, the greater the 


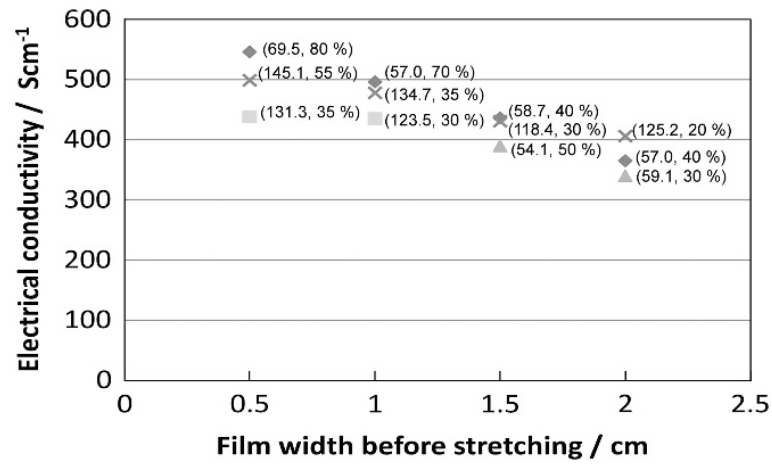

Figure 2 Relation between the film width before stretching and the electrical conductivity of the stretched PEDOT-PSS composite films containing xylitol (50 wt.\% xylitol of PEDOT-PSS). The thickness and stretching percentage of the stretched films are shown in parentheses. A full color version of this figure is available at Polymer Journal online.

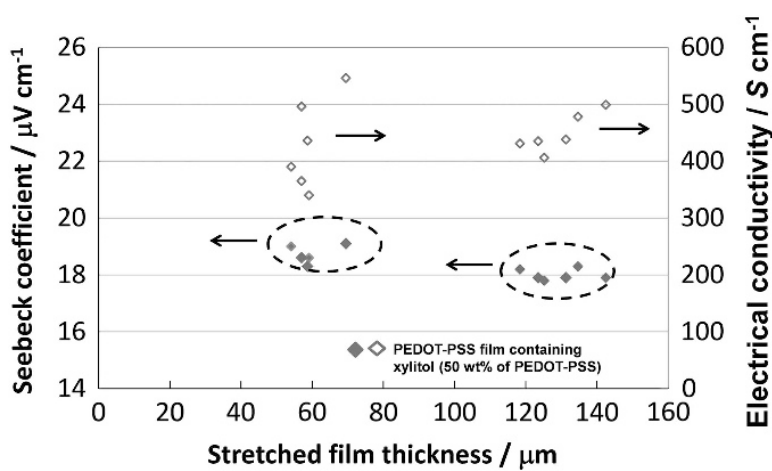

Figure 3 Relation between the stretched film thickness and the Seebeck coefficient (closed diamond) or the electrical conductivity (open diamond) of PEDOT-PSS films containing xylitol (50 wt.\% of PEDOT-PSS). A full color version of this figure is available at Polymer Journal online.

stretching percentage, the higher the electrical conductivity was for the rather narrow films, as shown in Figure 2. The results are consistent with those shown in Figure 1. There was a tendency for thinner films to have a higher electrical conductivity, as occurred in the case of narrow films that were $0.5 \mathrm{~cm}$ and $1.0 \mathrm{~cm}$ in width. This was not the case in wider films. Second, there was a slight correlation between the thickness and Seebeck coefficient of the stretched films and no correlation between the thickness and electrical conductivity at all, as shown in Figure 3. Third, Figure 4 shows that the electrical conductivity of the stretched composite film was clearly proportional to the stretching percentage, though little change can be observed in the Seebeck coefficient.

\section{Improvement of thermoelectric properties}

Table 1 summarizes the thermoelectric properties of the PEDOT-PSS films examined in this research: the pristine film (PEDOT-PSS, no. 1), the film prepared by the addition of DMSO (PEDOT-PSS(DMSO), no. 2), the composite film with xylitol (PEDOT-PSS(xylitol), no. 3), and the composite film prepared by the addition of DMSO (PEDOT-PSS(DMSO+xylitol), no. 4). The composite films PEDOTPSS(xylitol) and PEDOT-PSS(DMSO+xylitol) rinsed with water are also shown as PEDOT-PSS(xylitol)+rinsed (no. 5) and PEDOT-PSS (DMSO+xylitol)+rinsed (no. 6), respectively. Table 2 shows the

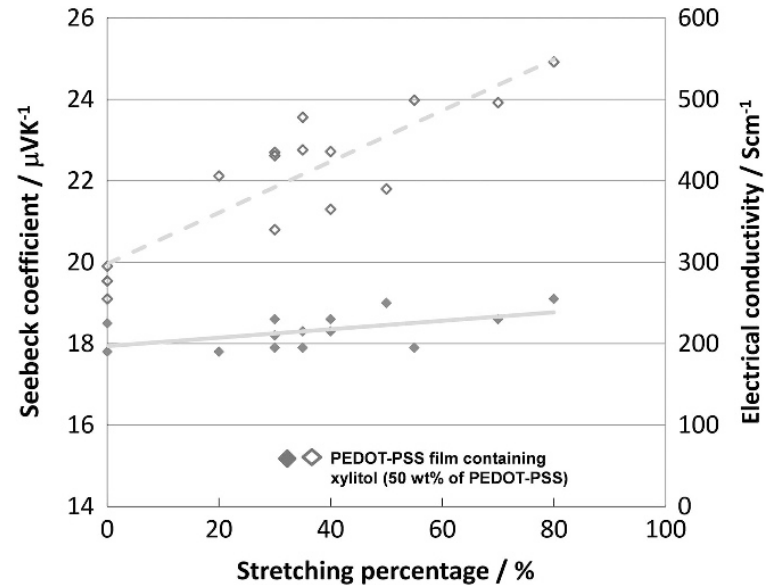

Figure 4 Relation between the stretching percentage and the Seebeck coefficient (closed diamond) or the electrical conductivity (open diamond) of PEDOT-PSS films containing xylitol (50 wt.\% of PEDOT-PSS). A full color version of this figure is available at Polymer Journal online.

estimated thermal conductivity, $\kappa$, of the PEDOT-PSS(xylitol) film to be $0.234 \pm 0.009 \mathrm{~W} \mathrm{~m}^{-1} \mathrm{~K}$, based on the data of $\alpha, \rho$ and $C_{\mathrm{p}}$.

It is known that PEDOT-PSS films that are prepared by the addition of DMSO, abbreviated as PEDOT-PSS(DMSO) in this paper, have a much higher electrical conductivity and a slightly lower Seebeck coefficient than do those prepared without DMSO. ${ }^{14-16}$ In fact, as shown in Table 1. PEDOT-PSS(DMSO) films have an electrical conductivity and Seebeck coefficient of 500-600 S cm $\mathrm{cm}^{-1}$ and $\sim 15 \mu \mathrm{V} \mathrm{K}^{-1}$, respectively, although the values depend on the thickness of the film. These values are much higher for the electrical conductivity and slightly lower for the Seebeck coefficient compared with those of the pristine film, as shown for film no. 1 in Table 1 (which has an electrical conductivity of $4.03 \pm 0.68 \mathrm{Scm}^{-1}$ and a Seebeck coefficient of $18.2 \pm 0.5 \mu \mathrm{V} \mathrm{K}^{-1}$ ). According to equations (1) and (3), the PEDOT-PSS(xylitol) films, which were prepared by the addition of $50 \mathrm{wt} . \%$ xylitol instead of $5 \mathrm{wt}$.\% DMSO, were expected to have a lower electrical conductivity and a higher Seebeck coefficient value than the pristine PEDOT-PSS films because the carrier concentration, which can be diluted by the insulating xylitol, is inversely proportional to the Seebeck coefficient and proportional to the electrical conductivity, based on these equations. However, the results shown in Table 1 are not consistent with the expectation. The significantly high electrical conductivity could be understood by considering that xylitol, a type of polyol, may work like DMSO or ethylene glycol. ${ }^{14-16}$

Because the addition of DMSO can increase the electrical conductivity and because the addition of xylitol can improve the Seebeck coefficient compared with that of PEDOT-PSS(DMSO), PEDOT-PSS films, including both DMSO and xylitol, were prepared for examination. Unfortunately, those films (no. 4 in Table 1) did not provide a good result. In contrast, when PEDOT-PSS(xylitol) films were rinsed with water (no. 5 in Table 1) or treated with DMSO and then rinsed with water (no. 6 in Table 1), the improvement of the Seebeck coefficient alone or of both the Seebeck coefficient and the electrical conductivity was achieved, as shown in Table 1.

Meanwhile, because stretching is a useful technique to improve the electrical conductivity ${ }^{10}$ and because the PEDOT-PSS(xylitol) films are stretchable, the stretching of PEDOT-PSS(xylitol) films was carried out to improve the thermoelectric properties. The films showed 
Table 1 Thermoelectric properties of PEDOT-PSS films with/w.o. 5 wt.\% DMSO and/or 50 wt.\% xylitol

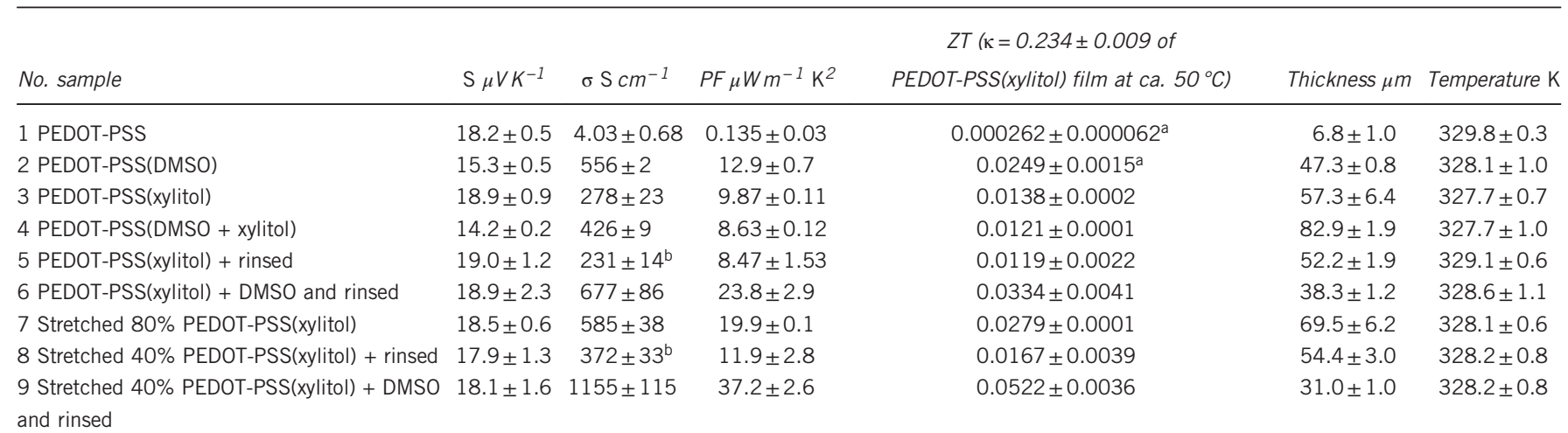

Abbreviations: DMSO, dimethyl sulfoxide; PEDOT-PSS, poly(3,4-ethylenedioxythiophene) poly(4-styrenesulfonate).

$\mathrm{a}_{\kappa}=0.17 \pm 0.01$ of PEDOT-PSS(DMSO) film at ca. $50^{\circ} \mathrm{C}$.

bMeasured by Lorester.

Table 2 Data for $\kappa$ estimate of PEDOT-PSS(xylitol) films

\begin{tabular}{lcccc}
\hline Sample & Thickness $\mu \mathrm{m}$ & $\alpha \mathrm{mm}^{2} \mathrm{~s}^{-1}\left(\mathrm{ca} .50^{\circ} \mathrm{C}\right)$ & $\rho \mathrm{gcm} \mathrm{cm}^{-3}$ (room temperature) & $\mathrm{C}_{\rho} \mathrm{Jg}^{-1} \mathrm{~K}\left(\mathrm{ca} .50^{\circ} \mathrm{C}\right)$ \\
\hline $3-1$ & $186 \pm 8$ & $0.114 \pm 0.004$ & $1.47 \pm 0.06$ & $1.40 \pm 0.04$ \\
$3-2$ & $53.1 \pm 2.1$ & $0.103 \pm 0.005$ & $1.62 \pm 0.07$ & $0.234 \pm 0.025$ \\
\hline
\end{tabular}

$C_{\mathrm{p}}$ value was measured for the PEDOT-PSS(xylitol) film with $82.0 \mu \mathrm{m}$ thick.

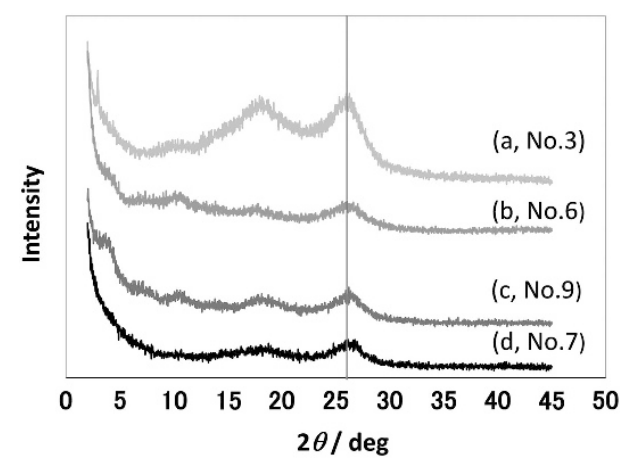

Figure 5 X-ray diffraction patterns of our PEDOT-PSS(xylitol) films: (a), the film without treatment, no. 3. (b), the film without stretching and with DMSO and rinsing, no. 6. (c), the film with stretching with treatment with DMSO and water, no. 9. (d), the film with stretching and without DMSO treatment, no. 7. A full color version of this figure is available at Polymer Journal online.

similar electrical conductivity values to PEDOT-PSS(DMSO) without decreasing the Seebeck coefficient, as shown in no. 7 in Table 1. A similar improvement in the electrical conductivity was observed by comparing no. 8 with no. 5 and no. 9 with no. 6 in Table 1 . These improvements in the electrical conductivity are attributed to the improvement in the alignment of the polymer chains. ${ }^{9,12}$ X-ray diffraction spectra were measured, as shown in Figure 5. The results are not very clear, but a small shift of the peaks to larger $2 \theta$ values can be detected in samples nos. 7 and 9 compared with those of nos. 3 and 6 , respectively, which may suggest a slight improvement in the alignment by shortening the intermolecular distance. To clarify the difference in X-ray diffraction patterns, the full-width at-halfmaximums of those four diffraction peaks at $2 \theta \sim 26^{\circ}$ were analyzed, and the crystal sizes based on the corresponding peaks were calculated using the Scherrer equation. The results show that the peaks of nos. 3, 6, 7 and 9 had full-width at-half-maximums of $0.444,0.444,0.356$ and 0.311 degrees, which correspond to crystal sizes of $33.5,33.5,41.8$ and $47.9 \mathrm{~nm}$, respectively. Hence, the crystal sizes of the stretched PEDOT-PSS(xylitol) composite films (nos. 7 and 9) were greater than those of the non-stretched films (nos. 3 and 6). It was discovered that stretching the films improved the polymer alignment and enlarged the crystalline parts of the polymer, which resulted in an increase in the electrical conductivity of the films.

The effect of treatment with both DMSO and water on the electrical conductivity of the composite films is clearly shown in Table 1 as a comparison of the data of nos. 8 and 9 with those of no. 7 . In the $\mathrm{X}$-ray diffraction patterns, all of the spectra in Figure 5 have peaks at $2 \theta \sim 17.5^{\circ}$ and $26^{\circ}$. The former peak comes from PEDOT, and the latter comes from PSS. ${ }^{18}$

Although the X-ray diffraction pattern difference in Figure 5 is not clear, a small shift to larger angles may be detected. Thus, the distance between PEDOT rings could be slightly shortened by stretching, which would result in a small shift to a higher angle in these peaks. In contrast, nos. 6 and 9 that contain DMSO, have peaks at $2 \theta \sim 4^{\circ}$ and $11^{\circ}$, which might imply that the DMSO works to change the crystal structure and results in an improvement of the PEDOT molecular alignment. To clarify the DMSO and water treatment effect, the crystalline sizes calculated from the X-ray peaks were compared between samples no. 3 (crystal size $33.5 \mathrm{~nm}$ ) and no. 6 (crystal size $33.5 \mathrm{~nm}$ ) among the non-stretched films and between samples no. 7 (crystal size $41.8 \mathrm{~nm}$ ) and no. 9 (crystal size $47.9 \mathrm{~nm}$ ) among the stretched films. There is no difference between the non-stretched samples no. 3 and 6 , but there is a clear difference between the stretched samples no. 7 and no. 9. Thus, in the non-stretched samples nos. 3 (crystal size $33.5 \mathrm{~nm}$ ) and 6 (crystal size $33.5 \mathrm{~nm}$ ), the increase in the electrical conductivity owing to the treatment with DMSO and water might be caused not by the improvement of the polymer 
alignment but rather by the removal of the insulating PSS polymer. In the stretched samples no. 7 and no. 9, however, the DMSO and water treatment clearly had an effect on the crystalline size, that is, the polymer alignment. This implies that not only the removal of the insulating polymer but also the alignment of the polymer chains caused by the DMSO and water treatment in the stretched films could cause an increase in the electrical conductivity and result in an improvement in the thermoelectric performance.

\section{CONCLUSION}

It was confirmed that adding xylitol to PEDOT-PSS films instead of DMSO resulted in flexible composite films, and the stretching technique was first applied to the PEDOT-PSS composite films to improve the thermoelectric properties. The Seebeck coefficient of the xylitol-containing PEDOT-PSS films was higher than those observed in films prepared by the addition of DMSO, which was likely due to the decrease in the carrier concentration of the PEDOT-PSS films that was caused by mixing with the insulating xylitol. Nevertheless, the electrical conductivity of the composite films was improved by stretching the films, which kept the Seebeck coefficient high, probably due to the increase in carrier mobility that resulted from the improvement of the polymer chain alignment. Furthermore, treatment with DMSO and pure water was successfully applied to the stretched PEDOT-PSS(xylitol) composite films to improve the electrical conductivity to approximately twice that of the pristine PEDOT-PSS (xylitol) composite films. The stretched and rinsed films had high electrical conductivity and thermoelectric power factor values of $1155 \pm 115 \mathrm{~S} \mathrm{~cm}^{-1}$ and $37.2 \pm 2.6 \mu \mathrm{W} \mathrm{m}^{-1} \mathrm{~K}^{-2}$, respectively. The cause for this could be the increase in the carrier concentration and carrier mobility by losing the excess insulating PSS from the treatment. ${ }^{19}$

Herein, compared with the thermoelectric properties of other PEDOT derivatives ${ }^{20}$ the performance of our stretched PEDOT-PSS (xylitol) composite films lies between that of PEDOT-PSS with and without diethylene glycol and that of PEDOT-Tos (tosylate) with and without N,N-dimethylformamide. In addition, it was reported that two main trends can be distinguished: $S$ is almost constant for all PEDOT-PSS samples, despite the large variation in $\sigma$, and $S$ increases markedly for highly conducting PEDOT-Tos samples, up to $55 \mu \mathrm{V} \mathrm{K}^{-1}$ for $1500 \mathrm{~S} \mathrm{~cm}^{-1}$. Our stretched PEDOT-PSS (xylitol) composite films illustrate the former trend.

Although the exact explanation of the effects of stretching and treatment with DMSO and water on the conducting PEDOT-PSS (xylitol) composite films is still ambiguous, these techniques could practically provide improved organic thermoelectric materials.

\section{CONFLICT OF INTEREST}

The authors declare no conflict of interest.

\section{ACKNOWLEDGEMENTS}

The present research was financially supported by the Regional Innovation Cluster Program (Global type), 'Yamaguchi Green Materials Cluster,' from the Ministry of Education, Culture, Sports, Science and Technology

(MEXT), Japan.

1 Toshima, N. Conductive polymers as a new type of thermoelectric materials. Macromol Symp. 186, 81-86 (2002).

2 Toshima, N. Recent progress of organic and hybrid thermoelectric materials. Kobunshi (Polymers) 63, 782 (2014).

3 Toshima, N. \& Ichikawa, S. Conducting polymers and their hybrids as organic thermoelectric materials. J. Electronic Mater. 44, 384-390 (2015).

4 Shirakawa, H., Louis, E. J., MacDiarmid, A. G., Chiang, C. K. \& Heeger, A. J. Synthesis of electrically conducting organic polymers: Halogen derivativrs of polyacetylene, $(\mathrm{CH})_{x}$. J. Chem. Soc. Chem. Comm. 1977, 578-580 (1977).

5 Okuzaki, H. in PEDOT: Material Properties and Device Applications. (ed. Okuzaki H.) (Science \& Technology, Ltd, Tokyo, Japan, 2012).

6 Louwet, F., Groenendaal, L., Dhaen, J., Manca, J., Van Luppen, J., Verdonck, E. \& Leenders, L. PEDOT/PSS: Synthesis, characterization, properties and applications. Synth. Met. 135-136, 115-117 (2003).

7 Yan, H. \& Toshima, N. Thermoelectric properties of alternatively layered films of polyaniline and $( \pm)$-10-camphorsulfonic acid-doped polyaniline. Chem. Lett. 1999, 1217-1218 (1999).

8 Yan, H., Sada, N. \& Toshima, N. Thermal transporting properties of electrically conductive polyaniline films as organic thermoelectric materials. J. Thermal Anal. Calorimetry 69, 881-887 (2002).

9 He, H. X., Li, C. Z. \& Tao, N. J. Conductance of polymer nanowires fabricated by a combined electrodeposition and mechanical break junction method. Appl. Phys. Lett. 78, 811-813 (2001).

10 Yan, H., Ohta, T. \& Toshima, N. Stretched polyaniline films doped by $( \pm)-10$ camphorsulfonic acid: Anisotropy and improvement of thermoelectric properties. Macromol. Mater. Eng. 286, 139-142 (2001).

11 Terasaki, I. in Handbook of Thermoelectric Conversion Technology. (ed. Kajikawa T.) Ch 2.1., 17-20 (NTS, Ltd, Tokyo, Japan, 2008).

12 Hiroshige, Y., Ookawa, M. \& Toshima, N. Thermoelectric figure-of-merit of iodine-doped copolymer of phenylenevinylene with dialkoxyphenylenevinylene. Synth. Met. 157, 467-474 (2007).

$13 \mathrm{Li}$, Y., Masuda, Y., Iriyama, Y. \& Okuzaki, H. Stretching and highly conductive polymer films. Trans. Mater. Res. Soc. Jpn. 37, 303-306 (2012).

$14 \mathrm{Kim}$, J. Y., Jung, J. H., Lee, D. E. \& Joo, J. Enhancement of electrical conductivity of poly(3,4-ethylenedioxythiphene)/poly(4-styrenesulfonate) by a change of solvents. Synth. Met. 126, 311-316 (2002).

15 Ashizawa, S., Horikawa, R. \& Okuzaki, H. Effects of solvent on carrier transport in poly(3,4-ethylenedioxythiophene)/poly(4-styrenesulfonate). Synth. Met. 153, 5-8 (2005).

16 Wei, Q., Mukaida, M., Naitoh, Y. \& Ishida, T. Morphological change and mobility enhancement in PEDOT:PSS by adding co-solvents. Adv. Mater. 25, 2831-2836 (2013).

17 DeLongchamp, D. M., Vogt, B. D., Brooks, C. M., Kano, K., Obrzut, J., Richter, C. A. Kirillov, O. A. \& Lin, E. K. Influence of a water rinse on the structure and properties of poly(3,4-ethylene dioxythiophen):poly(styrene sulfonate) films. Langmuir 21 11480-11483 (2005)

18 Chiang, C-H. \& Wu, C-G. High-efficient Dye-sensitized solar cell based on highly conducting and thermally stable PEDOT:PSS/glass counter electrode. Org. Electronics 14, 1769-1776 (2013)

19 Kim, G-H., Shao, L., Zhang, K. \& Pipe, K. P. Engineered doping of organic semiconductors for enhanced thermoelectric efficiency. Nat. Mater. 12 , 719-723 (2013).

20 Bubnova, O., Khan, Z. U., Wang, H., Braun, S., Evans, D. R., Fabretto, M., Hojati-Talemi, P., Dagnelund, D., Arlin, J-B., Geerts, Y. H., Desbief, S. Breiby, D. W., Andreasen, J. W., Lazzaroni, R., Chen, W. M., Zozoulenko, I., Fahlman, M., Murphy, P. J., Berggren, M. \& Crispin, X. Semi-metallic polymers. Nat. Mater. 13, 190-194 (2014). 